\title{
Frisby Davis distance stereoacuity values in visually normal children
}

\author{
W E Adams, S Hrisos, S Richardson, H Davis, J P Frisby, M P Clarke
}

Br J Ophthalmol 2005;89:1438-1441. doi: 10.1136/bjo.2005.071761

Aims: To establish the range of normal distance stereoacuity in young children using the Frisby Davis distance stereotest (FD2).

Methods: Children passing preschool vision screening assessments underwent measurement of distance stereoacuity with the FD2 using a standard testing protocol.

Results: 59 visually normal children aged between 36 months and 68 months were recruited to this study. All 59 were able to understand the test requirements and were examined with the FD2 stereotest. Four $(6.8 \%)$ had no measurable stereoacuity; $13(24 \%)$ had stereoacuity measurable only at a 3 metre testing distance (mean 92.3 seconds of arc; SD 52.6). These children were significantly younger than the remaining 42 (76\%) who demonstrated a stereoacuity response at a 6 metre testing distance (mean 29.6 seconds of arc; SD 13.1, $p=0.008$ ).

Conclusion: The FD2 stereotest enables the measurement of distance stereoacuity in young children. There appears to be a maturational effect with distance stereoacuity improving between 36 months and 68 months. The data on age related normal values will provide a baseline from which to compare outcomes in clinical populations.

S tereopsis is the perception of depth on the basis of binocular disparity. ${ }^{1}$ Tests of near stereoacuity-for example, TNO, Randot, Frisby, are used in the management of strabismus and amblyopia. The measurement of distance stereoacuity provides useful information regarding the management of strabismus primarily affecting distance fixation..$^{2-5}$

Measurement of stereopsis to distant targets is possible using the synoptophore, the AO Vectographic Project-OChart Slide test, and the MentorII-SG B-VAT (Baylor Video Acuity Test-Mentor system 2). The latter two tests require the patient to wear spectacles. The AO Vectographic Project-OChart Slide test uses polarising lenses on a phoropter, generating disparities from 480-30 seconds of arc. ${ }^{6}$ The Mentor II-SG B-VAT uses liquid crystal goggles and generates disparities from 240-15 seconds of arc. ${ }^{7}$

Most of the studies using these methods of distance stereopsis measurement have been performed on patients who are 5 years and older although one study used children 4 years and older. ${ }^{2-5}{ }^{8-12}$ As the majority of cases of paediatric strabismus present before the age of 5 years, these tests have had limited clinical application. No study to date has measured distance stereopsis in children as young as 3 years.

In an attempt to overcome this difficulty two of the authors (JPF and HD) invented the Frisby Davis distance stereotest (FD2) as a child friendly, free space test of real depth. ${ }^{13}$ We undertook to assess the ease with which this test could be delivered and to establish the range of values that could be achieved in young, visually normal children.

\section{PATIENTS AND METHODS \\ Patients}

Distance stereoacuity measurements were obtained from children undergoing preschool vision screening. The majority (57/59) of the children were white and as far as could be ascertained, developmentally normal.

All the children had their visual acuity tested at 6 metres, using the Sheridan Gardner test. The presence of binocular single vision was assessed by an orthoptist who tested the near and distance cover test, the Lang-II Stereotest, and 20 dioptre prism test.

Those who achieved normal visual acuity and binocular single vision were then assessed for distance stereoacuity, using the FD2, by an independent examiner.

\section{Methods}

The FD2 test comprises a box containing four back illuminated and differently shaped plastic objects mounted on rods.

These are either four animal or four geometric shapes set in a transparent frame pointing towards the observer (fig 1).

The shapes are translucent but sufficiently dark to obscure the rods, giving the appearance that the shapes are free floating. One shape is set by the examiner to be nearer to the observer at each presentation and the test requirement is to identify this target. The size of disparity presented is altered by the amount by which the rod is set to protrude and by the distance of the observer from the targets, generating disparities from 200-4 seconds of arc. ${ }^{14}$

A testing protocol was written to establish comprehension of the test and to standardise the testing procedure (fig 2).

Comprehension of the test was established by asking the child, at 1 metre distance from the box, to name the animal shapes. One shape was set to protrude and the child was asked to identify which animal "jumped out" of the box. The testing was then performed starting with the largest disparity at 6 metres. If the child could not respond accurately at 6 metres then the distance was reduced to 3 metres and the procedure repeated. Recorded stereoacuity values took account of the test distance.

There were three possible outcomes on the test, recorded as follows:

(1) Non-responder: unable to comprehend the test when presented with the largest disparity at 1 metre.

(2) Negative: comprehension established at 1 metre but incorrect responses for two presentations at the largest disparity at 6 metres and then also at 3 metres.

(3) Responder: comprehension established and stereoacuity measured to threshold, either at 6 metres or 3 metres.

Abbreviations: FD2, Frisby Davis distance stereotest 


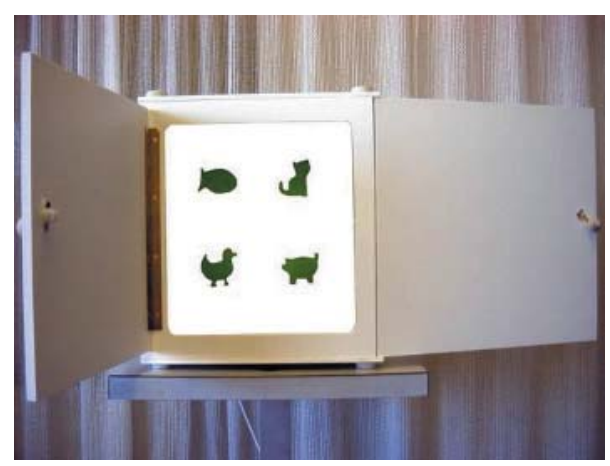

Figure 1 FD2 stereotest using animal shapes. The animal shapes were used in this study.

Mean stereoacuity values were calculated for the group as a whole and then subdivided for those performing at 6 metres and at 3 metres. The data were examined for a correlation between age and level of stereoacuity achieved using Pearson product moment correlation.

\section{RESULTS}

Fifty nine children were examined, 34 male and 25 female. Mean age (SD) at testing was 47.7 (7.6) months (range 3668 months). No child was classified a non-responder.

Table 1 summarises the data for the whole study group: $4 / 59(6.8 \%)$ could comprehend but could not complete the test at 6 metres or 3 metres and were therefore classified as FD2 negative (age range 39-64 months).
The mean (SD) stereoacuity of the 55 responders was 44.5 (38.3) seconds of arc. Of these $42(76 \%)$ were able to perform the test at 6 metres and $13(24 \%)$ at 3 metres. The mean stereoacuity (SD) of those tested at 6 metres was 29.6 (13.1) seconds of arc and for those tested at 3 metres was 92.3 (52.6).

Children who were only able to complete the test at 3 metres were significantly younger than those who performed the test at 6 metres $(t=2.746, \mathrm{df}=53, \mathrm{p}=0.008$, two tailed). To maintain the integrity of the data, "6 metre failures" were excluded from further analysis.

A statistically significant relation between age and FD2 stereoacuity is still apparent in the group successfully performing the test at 6 metres, as shown in figure 3 (Pearson product moment correlation coefficient: -0.473 , $\mathrm{p}<0.002$, two tailed $\mathrm{n}=42$ ).

Table 2 shows the suggested age related normal values for those tested at 6 metres. Values have been rounded to the nearest measurable stereoacuity threshold. The values presented for the younger children are tentative as they are limited by the small sample.

\section{DISCUSSION}

The data presented in this study show that children as young as 39 months, with normal vision and ocular alignment, are easily able to perform the FD2 distance stereoacuity test.

As far as we are aware this is the first commercially available distance stereoacuity test that can be utilised in this age group.

Despite demonstrating comprehension of the test at 1 metre in all the children, there were a small number who

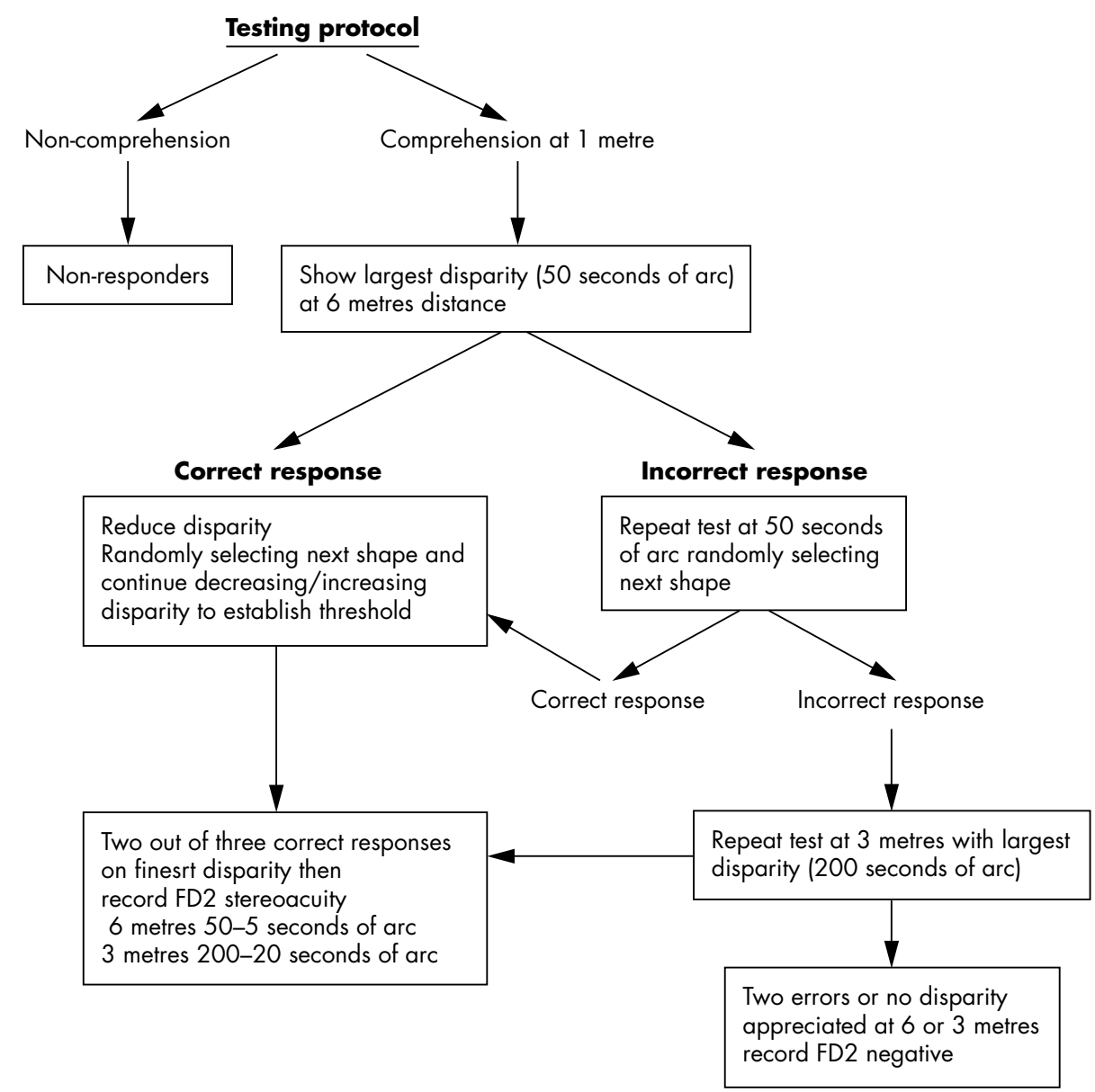

Figure 2 Testing protocol. 
Table 1 Summary of FD2 data for all children examined

\begin{tabular}{|c|c|c|c|c|c|}
\hline \multirow[b]{2}{*}{ Study status } & \multirow{2}{*}{$\begin{array}{l}\text { Mean age (SD) } \\
\text { (months) }\end{array}$} & \multicolumn{2}{|l|}{ Sex } & \multirow{2}{*}{$\begin{array}{l}\text { Mean stereopsis } \\
\text { (seconds of arc) } \\
\text { (SD) }\end{array}$} & \multirow[b]{2}{*}{ No } \\
\hline & & $M$ & $\mathbf{F}$ & & \\
\hline Responders & $47.7(7.6)$ & 31 & 24 & $29.6(13.1)$ & 55 \\
\hline 6 metres & $49.2(7.9)$ & 22 & 20 & $92.3(52.6)$ & 42 \\
\hline 3 metres & $42.9(3.9)$ & 7 & 6 & & 13 \\
\hline FD2 negative & $47.8(11.1)$ & 3 & 1 & & 4 \\
\hline Total number & & 34 & 25 & & 59 \\
\hline
\end{tabular}

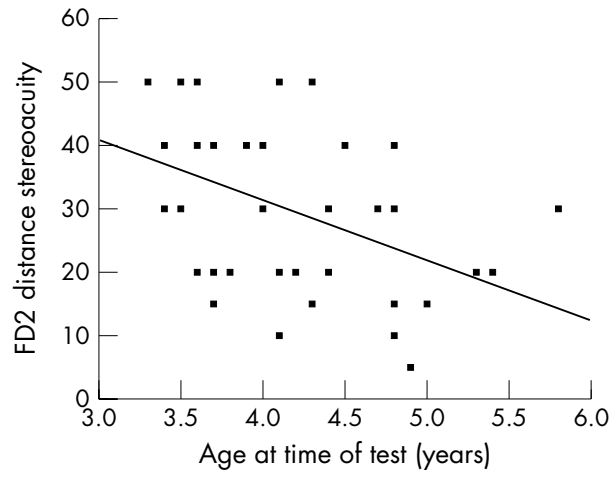

Figure 3 Relation between FD2 stereoacuity and age in those children who completed the test at 6 metres.

Table 2 Suggested FD2 stereoacuity norms (seconds of arc) (SD) for children $\geqslant 48$ months and $<48$ months performing the test at 6 metres.

\begin{tabular}{ll}
\hline Mean age & $\begin{array}{l}\text { Mean stereoacuity (seconds of arc) } \\
\text { at } 6 \text { metres test distance (SD) }\end{array}$ \\
\hline Age $\geqslant 48$ months $(n=29)$ & $30(13)$ \\
Age $<48$ months $(n=13)$ & $50(8)$ \\
Total number & 42 \\
\hline
\end{tabular}

could not respond at either of the set test distances (negative responders). It is possible that these children had defective distance stereoacuity but it is our impression that they failed because of an inability to cope with the cognitive challenges of testing - that is, attention, concentration, etc, rather than because they possess a less mature stereoscopic vision system. Stereoacuity arises between 3 months and 5 months of age but does not reach adult levels until around 3-7 years, depending on which test is used, and such challenges are therefore common when testing young children. ${ }^{15-19}$

Consistent with this we found that performance on the test was influenced by age-that is, younger children often showed poorer responses at 6 metres but responded well at 3 metres.

The FD2 is constructed as a real depth, free space test enabling assessment of stereoacuity without dissociating the eyes. This type of construction reflects binocular viewing as it occurs in everyday life but there is the potential to allow positive responses based on perception of monocular cues.

The test was developed to keep such cues to a minimum, as described in the FD2 test booklet; however, a recent study on monocular cues, using the FD2, in older patients has led its authors to propose a revised test protocol introducing a monocular test phase after binocular testing is complete. ${ }^{14} 20$

When using the standard FD2 testing protocol, young children do not seem susceptible to monocular cues, but it remains a possibility and could have resulted in an overestimation of performance. The application of the FD2 test in clinical practice is the subject of an ongoing study.

The suggested age related normal values in this study provide a useful reference from which to compare data in clinical populations. While this study sample is small, no other study to date has provided normal data in this age group. It is envisaged that the FD2 will prove a useful tool in the evaluation of binocular status, especially in intermittent exotropia.

Work has already been undertaken in patients with intermittent exotropia using the BVAT mentor, some of which suggests that measurements of decreasing distance stereoacuity may provide an indication for surgical intervention..$^{3-5} 11$

Studies are under way using the FD2 in patients with intermittent exotropia and results are awaited from them.

\section{CONCLUSION}

The FD2 stereotest enables the measurement of distance stereoacuity in young children; however, there appears to be a developmental effect of age on distance stereoacuity. The reported data presented here on age related normal values, will provide a baseline from which to compare outcomes in clinical populations.

\section{Authors' affiliations}

W E Adams, S Richardson, Department of Ophthalmology, Royal Victoria Infirmary, Newcastle upon Tyne, UK

S Hrisos, Research Associate, Centre for Health Services Research, University of Newcastle upon Tyne, Newcastle upon Tyne, UK

M P Clarke, Department of Ophthalmology, University of Newcastle upon Tyne, Newcastle upon Tyne, UK

H Davis, Academic Unit of Ophthalmology and Orthoptics, University of Sheffield, Sheffield, UK

J P Frisby, Department of Psychology, University of Sheffield, Sheffield, UK

Competing interests: John P Frisby and Helen Davis invented the FD2 stereo acuity test. The FD2 stereoacuity test is marketed via Stereotest Ltd. JP Frisby is the director of this company. The other authors have no competing interest.

Ethical approval was provided by Newcastle upon Tyne local ethics committee to use the FD2 as an adjunct to preschool vision screening.

Correspondence to: Wendy E Adams, Royal Victoria Infirmary, Queen Victoria Road, Newcastle upon Tyne NE1 4LP, UK; wendy_stoer@ yahoo.com

Accepted for publication 25 June 2005

\section{REFERENCES}

1 Bishop PO. Binocular Vision. In: Moses RA, Hart W M, eds. Adler's physiology of the eye: clinical application. St Louis: CV Mosby, 1987:619-89.

2 Zanoni D, Rosenbaum AL. A new method for evaluating distance stereoacuity. J Pediatr Ophthalmol Strabismus 1991;28:255-60.

3 O'Neal TD, Rosenbaum AL, Stathacopoulos RA. Distance stereoacuity improvement in intermittent exotropic patients following strabismus surgery. J Paediatr Ophthalmol Strabismus 1995;32:353-7. 
4 Stathacopoulos RA, Rosenbaum AL, Zanoni D, et al. Distance stereoacuity. assessing control in intermittent exotropia. Ophthalmology 1993; 100:495-500.

5 Yildirim C, Altinsoy HI, Yakut E. Distance stereoacuity norms for the mentor BVAT II-SG video acuity tester in young children and young adults. J AAPOS 1998;2:26-32.

6 Pratt-Johnson JA, Tillson G. Management of strabismus and amblyopia: a practical guide. New York: Thieme Medical, 1994:39.

7 Instruction manuals: B-VAT II-SG Video Acuity Tester. Norwell, MA: Mentor $\mathrm{O} \& \mathrm{O}, 1987$

8 Mehta AM, France TD. Distance stereoacuity in children and teenagers with normal near stereoacuity. Am Orthopt J 1997;47:144-7.

9 Yildirim C, Mutlu FM, Chen Y, et al. Assessment of central and peripheral fusion and near and distance stereoacuity in intermittent exotropic patients before and after strabismus surgery. Am J Ophthalmol 1999;128:222-30.

10 Rutstein RP, Corliss DA. Distance stereopsis as a screening device. Optom Vis Sci 2000;77:135-9.

11 Rutstein RP, Corliss DA. BVAT Distance vs near stereopsis screening of strabismus, strabismic amblyopia and refractive amblyopia; a prospective study of 68 patients. Binocul Vis Strabismus Q 2000;15:229-336.
12 Parks MM. The monofixation syndrome. Trans Am Ophthalmol Soc 1969;67:609-57.

13 Davis H, Frisby JP, Walters BC. The Frisby Davis distance stereotest (FD2). In: de Faber J-T, ed. Transactions of 27th Meeting European Strabismological Association. Lisse: Swets and Zeitlinger, 2001:33-6.

14 Frisby Davis Distance (FD2) Stereotest Booklet. Test design and test instructions.

15 Birch EE. Stereopsis in infants and its developmental relation to visual acuity. In: Simons K, ed. Early visual development: normal and abnormal. New York: Oxford University Press, 1993:224-36.

16 Fox R, Patterson R, Francis EL. Stereoacuity in young children. Invest Ophthalmol Vis Sci 1986;27:598-600.

17 Heron G, Dholakia S, Collins DE, et al. Stereoscopic threshold in children and adults. Am J Optom Physiol Opt 1985;62:505-15.

18 Simons K. Stereoscopic norms in young children. Arch Ophthalmol 1981;99:439-45.

19 Walraven J, Janzen P. TNO stereopsis test as an aid to the prevention of amblyopia. Ophthalmic Physiol Opt 1993;13:350-6.

20 Holmes JM, Fawcett S. Testing distance stereoacuity with the Frisby-Davis 2 (FD2) test. Am J Ophthalmol 2005;139:193-5. 